\title{
An Efficient Pseudo-Random Generator Provably as Secure as Syndrome Decoding
}

\author{
Jean-Bernard Fischer ${ }^{1}$ and Jacques Stern ${ }^{2}$ \\ ${ }^{1}$ Thomson Consumer Llectronics R\&D France \\ Parc d'innovation $\mathrm{n}^{\circ} 1$, B.P. 120, 67403 Illkirch Cedex, lirance \\ fischerjotce-rdf.fr \\ ${ }^{2}$ Ecole Normale Supérieure, Laboratoire d'informatique: \\ 45, rue d'Ulm, 75230 Paris Cedex 05, France \\ Jacques. Sternoens. fr
}

\begin{abstract}
We show a simple and efficient construction of a pseudorandom generator based on the intractability of an $\mathrm{NP}$-complete problem from the area of error-correcting codes. The generator is proved as secure as a hard instance of the syndrome decoding problem. Each application of the scheme generates a linear amount of bits in only quadratic computing time.
\end{abstract}

\section{Introduction}

A pseudo-random generator is an algorithm producing strings of bits that look random. The concept of "randomly looking" has been formalized by Blum and Micali [4] within the framework of complexity theory. Yao [22] has shown that the existence of a one-way permutation is sufficient to construct a pseudo-random generator. Subsequently, a long series of deep articles led to the conclusion that the existence of a one-way function is equivalent to the hypothesis that a pseudorandom generator exists $[15,10,14]$. However, the theoretical constructions proposed in these articles are often impractical.

Several schemes have been proposed which have a "proven security", i.e. based on the difficulty of well known problems like factorization $[21,3,1,18]$ or the discrete $\operatorname{logarithm}[4,16,12]$. But these propositions suffer from a relatively slow computing rate (i.c. they need much computation per generated bit). For example, outputting a single bit for the BBS generator takes quadratic time, and cubic time for the RSA based generators. This can be slightly enhanced to a. $\log _{2}(n)$ oulput.

'Therefore, it is interesting to study now schemes based on difficult problems not related to number theory. In an early work [9], Goldreich, Krawczyk and Luby established that the existence of a pseudo-random generator could be based on hard problems from the theory of crror-correcting codes. Unfortunately, their construction was a bit intricate. In the same vein, Impagliazzo and Naor [13] devised an elegant construction of a pseudo-random generator based on the subset-sum problem. Unfortunately, many rescarchers think that the underlying 
problem is computationnally rather weak. We present a new scheme bascd on the syndrome decoding problem, which is believed to be hard on most instances. Our scheme is extremely simple and achieves quadratic time with respect to the security parameters for producing a lincar arnount of random bits.

\subsection{The Syndrome Decoding problem}

In the field of crror correcting codes, one of the basic open problems is to find efficient algorithms for the decoding of random linear codes. Codes with a constant information rate and correcting a constant fraction of bits are particularly interesting.

A $(n, k, d)$ binary lincar code is a subspace of $\{0,1\}^{n}$ of size $2^{k}$ where every non zero word has weight at least $d$. It's information rate is $k / n$ and it can correct up to $\left\lfloor\frac{d-1}{2}\right\rfloor$ errors. It can be defined by its parity check matrix which is a $n$-by- $(n-k)$ binary matrix with the property that, for each vector of the code, the product $(\bmod 2)$ of the matrix by the vector is zero; this product can actually be computed for any vector and is called the syndrome. If the vector is not in the code, the syndrome is the sum of the columns of the matrix corresponding to positions where one bits are locatcd and is non zero.

Random linear codes are defined by a random parity check matrix. For such codes, no efficient algorithm is known for finding the closest code word to a vector, given its syndrome. It is also difficult to find a word of given weight from his syndrome's value [2]. This is called the syndrome decoding problem.

These problems are NP-complete. For further information, we refer to the books by McWilliams and Sloane [17] for error correcting codes, and by Garey and Johnson [7] for NP-complete problems. The syndrome decoding problem is NP-hard; see Berlekamp, McEliece and van Tilborg [2] for a proof. It can be stated as follows [7]:

Instance: An $m \times n$ binary matrix $A=\left(a_{i j}\right)$, a binary non-null vector $y=$ $\left(y_{1}, \ldots, y_{m}\right)$, and a positive integer $w$.

Question: Is there a binary vector $x=\left(x_{1}, \ldots, x_{n}\right)$ with no more than $w$ I's such that, for $1 \leq j \leq m, \sum_{i=1}^{n} x_{i} \cdot a_{i j} \equiv y_{j} \quad(\bmod 2)$ ?

Comment: The variant in which we ask for an $x$ with exactly $w$ 's is NPcomplete, even for $y=(0,0, \ldots, 0)$. If wo drop the word exactly, the question becomes open.

NP-hardness ensures that there is no polynomial-time algorithm for solving the problem in the worst casc; however many NP-completc problems can be efficiently solved in the average case. So we have to study the hardness of a random instance. This leads to practical issues that have been extensively studied by various people, as reviewed by Chabaud $[6,19]$. Algorithms known to solve the syndrome decoding problem are all probabilistic, so that we will speak about the probability of finding a word. They all have a computing complexity that grows exponentially in the size of $n$. They also give strong evidence that, in the case of 
random codes, the problem is the hardest for weights in the neighbourghood of the Gilbert-Warshamov bound corresponding to the dimensions of the code. The Gilbert-Warshamov bound $\lambda$ of a $(n, k, d)$ binary code is defined by the relation $1-k / n=H_{2}(\lambda)$ where $H_{2}(x)=-x \log _{2} x-(1-x) \log _{2}(1-x)$.

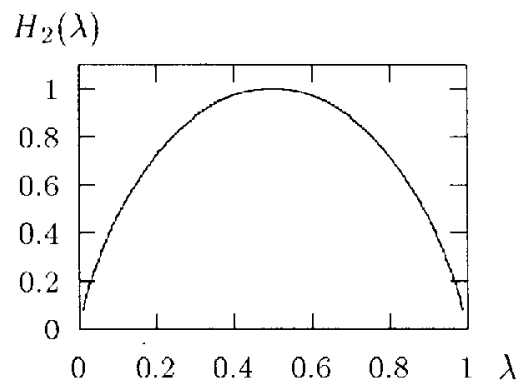

What comes out of the analysis is that it is relatively easy to find words of very low weight and given syndrome, but that the probability of finding one increases exponentially with the weight of the word (these algorithms are all probabilistic). However, the number of words having a given syndrome also grows exponentially with the weight; the point where there is an average of one word per syndrome value is the Gilbert-Warshamow bound. Finally, given a random vector with weight below the bound, the probability for it of having a pre-image is exponentially small in the weight.

We reach the following experimental conclusion: the difficulty of finding a word is a function of its weight that is growing until the Gilbert-Warshamov bound, and is then decreasing. So we can define a hard instance of syndrome decoding as an instance where the weight of the vectors is close to the GilbertWarshamov bound.

\subsection{Formal version of the intractability assumption}

In the sequel, we will only consider subsets $D_{n}$ such that, for suitable functions $k(n)$ and $l(n), D_{n}$ is included in $\{0,1\}^{l(n)}$ and is the one-one image of $\{0,1\}^{k(n)}$ by a polynomial-time function. This is a particular case of what is called polynomially-samplable [8].

We now define what we mean by hard (this is essentially a simplified version of Goldreich [8]):

Definition $1 A$ collection of functions $\left\{f_{n}: D_{n} \mapsto\{0,1\}^{k(n)}\right\}$ is called strongly one-way if the following two conditions hold:

there exists a polynomial-time algorithm $F$ that, on input $x \in D_{n}$, always outputs $f_{n}(x)$.

- for every probabilistic polynomial-time algorithm $A$, cvery $c>0$ and all sufficiently large $n$ 's,

$$
\operatorname{Pr}\left(A\left(f_{n}\left(X_{n}\right)\right) \in f_{n}^{-1} \int_{n}\left(X_{n}\right)\right)<\frac{1}{n^{c}}
$$


where $X_{n}$ is a random variable uniformely distributed over $D_{n}$.

We now consider a very general collection of functions related to the syndrome decoding problem.

Definition 2 Let $\rho$ be in $] 0,1\left[;\right.$ lel $w$ and $w^{\prime}$ be two integer functions such that $w(n) \leq w^{\prime}(n)<n$. The $S D\left(\rho, w, w^{\prime}\right)$ collection is the set of functions $\left\{f_{n}\right\}$ such lhat:

$$
\begin{gathered}
D_{n}=\left\{(M, x), M \in\lfloor\rho n\rfloor \times n, x \in\{0,1\}^{n} / w(n) \leq|x| \leq w^{\prime}(n)\right\} \\
f_{n}: D_{n} \longrightarrow\{0,1\}^{\lfloor\rho n\rfloor \cdot(n+1)} \\
(M, x) \longmapsto(M, M \cdot x)
\end{gathered}
$$

As we have seen in the previous section, instances of the problem where $w(n)$ and $w^{\prime}(n)$ are very small or close to $n / 2$ are not hard, so that we want to restrict the collection to the instances where the weight of $x$ is near the GilbertWarshamov bound. Clearly, it is not known if such a collection is one-way, but we have seen that, despite extensive research, no cfficient inversion algorithm has been found. So we make the assumption that the collection is one-way for weights slightly below the Gilbert-Warshamov hound.

Intractability assumption 1 Let $\rho$ be in $] 0,1\left[\right.$; let $\lambda$ be defined by $\rho=H_{2}(\lambda)$ and $\lambda<1 / 2$. Then, for any positive real $\epsilon$, if we set $w(n)=\left\lfloor\frac{\lambda}{1+\epsilon} n\right\rfloor$ and $w^{\prime}(n)=$ $\lfloor\lambda n\rfloor$, the $S D\left(\rho, w, w^{\prime}\right)$ collection is strongly one-way.

Note: $D_{n}$ has to be polynomially-samplable. We will discuss this point later on.

Of particular interest to us is the case where the weight of $x$ is fixed and is a constant fraction of $n$, i.e. $w(n)=w^{\prime}(n)=\lfloor\delta n\rfloor$ for some $\delta$ in $] 0,1[$. The corresponding intractability assumption reads as follows:

Intractability assumption 2 Let $\rho$ be in $] 0,1[$. Then, for all $\delta$ in $] 0,1 / 2$ [ such that $I_{2}(\delta)<\rho$, the $S D(\rho, \delta, \delta)$ collection of functions is strongly one-way.

We now write $S D(\rho, \delta)$ to refer to our second assumption with fixed parameters $\rho$ and $\delta$. We will occasionnally omit the parameters thus writing $S D$ in place of $S D(\rho, \delta)$. The corresponding function will be denoted by $f_{n}^{\rho, \delta}$ or simply $f_{n}$ and has domain $\left.D_{n}^{\rho, \delta}=\left\{(M, x), M \in\lfloor\rho n\rfloor \times n, x \in\{0,1\}^{n} /|x|=\delta\right)\right\}$

Cryptographic applications of the syndrome decoding problem have appeared in an identification scheme devised by the second named author [20].

Note that if $H_{2}(\lambda)=\rho$ and $\delta<\frac{\lambda}{2}$, the intractability of $S D(\rho, \delta)$ is a particular case of the difficulty of decoding below half the minimum distance. Thus our assumption is stronger than the usual decoding assumptions (see [9]). The construction that appears in section 2 can equally be based on the hardness of decoding but, when we come to practical issues, codes of a larger dimension will be needed. 'This is why we chose to work with our SD assumption. 


\section{A pseudo-random generator based on the syndrome decoding problem}

\subsection{Construction of the generator}

Our goal is to construct an efficient pseudo-random generator based on a hard problem, so that the generator inherits the hardness of the problem. We have defined a hard instance of the syndrome decoding problem and a collection of functions $S D(\rho, \delta)$ one-way under the second intractability assumption.

Let us first note that $f_{n}^{\rho, \delta}$ expands its input: the size of the input set is $2^{\lfloor\rho n\rfloor \cdot n} \cdot\left(\begin{array}{c}n \\ \delta n\end{array}\right)$, the size of the image sct is $2^{\lfloor\rho n\rfloor \cdot n} \cdot 2^{\lfloor\rho n\rfloor} ;$ since $H_{2}(\delta)<\rho$, there exists a positive real $\epsilon$ such that $(1+\epsilon) \log _{2}\left(\begin{array}{c}n \\ \delta n\end{array}\right)=\lfloor\rho n\rfloor$. 'That means that for a sufficiently large $n, f_{n}^{\rho, \hat{r}}$ expands its input by some linear amount of bits. We note also that $f_{n}^{\rho, \delta}$ can be computed in time $O\left(n^{2}\right)$, since it is exactly the binary product of a $\lfloor\rho n\rfloor \times n$ matrix with a $\lfloor\rho n\rfloor$ column vector.

We construct the generator $G_{\rho, \delta}$ in the following way:

Input: $(M, x) \in D_{n}^{p, \delta}$

Output: $\int_{n}^{p, \delta}(M, x)=(M, M \cdot x)$

Following a standard construction, we also consider an iterative generator $g_{\rho, \delta}$ that, on input $(M, x)$, outputs as many bits as we like. To perform this iteration, we need an efficient algorithm that computes a vector of size $n$ and weight $\delta$ from a $\log _{2}\left(\begin{array}{c}n \\ \delta n\end{array}\right)$ bit number. For the time being, we consider such an algorithm $A$ as granted and we will describe one in the next section. Wo get the following:

Input: $(M, x) \in D_{n}^{\rho, \delta}$

1. compute $y=M \cdot x$

2. separate $y$ in two bit strings $y_{1}$ and $y_{2}, y_{1}$ being the first $\left\lceil\log _{2}\left(\begin{array}{c}n \\ \delta n\end{array}\right)\right\rceil$ bits of $y$ and $y_{2}$ the romaining bits.

3. output $y_{2}$

4. set $x \leftarrow A\left(y_{1}\right)$ and goto 1 .

\subsection{An algorithm for generating all words of given weight}

The first and second intractability assumptions require a polynomial-time algorithm that samples the set of vectors of given weight. Such an algorithm is also needed in our scheme for the pscudo-random generator, but furthermore, it has to be computable in quadratic time to preserve the efficiency of the gencrator. 
We will use an algorithm inspired by Guillot [11]. This algorithm is said quadratic, takes as input a word of exactly $\log _{2}\left(\begin{array}{l}n \\ w\end{array}\right)$ bits and outputs a word of length $n$ and weight $\lfloor\delta n\rfloor$.

Ilowever, in his paper, Guillot uses a heuristic approximation to justify the computing time $O\left(n^{2}\right)$. He assumes that multiplication and division of a large number (of size $n$ ) by a small number (of $\operatorname{size} \log _{2} n$ ) are linear because the small number has typically the size of a computer word. This argument is not enough for a more formal approach.

In the Turing machine model, the algorithm is "almost" quadratic since it has a of complexity $O\left(n^{2} \log n\right)$. However the quadratic time bound holds in the model corresponding to random machines with logarithmic cost. See the appendix for a proof.

We now present the algorithm for gencrating words of given weight. It is based on an efficient way of outputting a word of length $n$ and weight $w$ given its index in the lexicographic ordering (see [11]).

We write the lexicographic enumeration algorithm in the following way:

Input: $i, n, w$

1. $c \leftarrow\left(\begin{array}{l}n \\ w\end{array}\right)$

2. while $n>0$ do
(a) $c^{\prime} \leftarrow c \cdot \frac{n-w}{n}$
(b) if $i \leq c^{\prime}$
- output 0
$-c \leftarrow c^{\prime}$
(c) clse
- output 1
$-i \leftarrow i-c^{\prime}$
$-c \leftarrow c \cdot \frac{w}{n}$
(d) $n \leftarrow n-1$

From the lemma, wo notc that the initial computation of $\left(\begin{array}{l}n \\ w\end{array}\right)$ takes quadratic time. The two cases in the while loop respectively take care of words where a zero (resp. a one) comes first: entering the loop with the initial values of the variables, we find $\left(\begin{array}{c}n-1 \\ w\end{array}\right)$ words starting with 0 , the remaining with 1 . The same happens in subsequent iterations, mutatis mutandis.

\subsection{Security of the scheme}

Goldreich and Levin [10] have shown that the inner product is a hard bit for any one-way function. Recall that, if $x, r \in\{0,1\}^{n}$, the inner product $r \odot x$ is the parity of the number of positions where the bits of $x$ and of $r$ are both 1 $\left(r_{i}=x_{i}=1\right)$. The hardness result reads as follows: 
Theorem 1 [10] Let $f: D_{n} \subset\{0,1\}^{l(n)} \rightarrow\{0,1\}^{k(n)}$ be a one-way function. For every polynomial-time algorithm $A$, cvery polynomial $p$ and all bul finitely many n's,

$$
\operatorname{Pr}(A(f(x), r)=r \odot x)<\frac{1}{2}+\frac{1}{p(n)}
$$

where the probatility is taken over uniformly chosen $x \in D_{n}$ and $r \in\{0,1\}^{l(n)}$.

An algorithm is a pseudo-random generator if its output distribution is polynomial-time indistinguishable from a truly random distribution. Two distributions $X$ and $Y$ are indistinguishable in polynomial-timo if, for cvery probabilistic polynomial-time algorithrn $D$ and every polynomial $p, \mid \operatorname{Prob}(D(x)=$ 1) $-\operatorname{Prob}(D(y)=1) \mid<\frac{1}{p(n)}$ where probabilities are taken over $X$ and $Y$. We will prove that the generator $G_{\rho, \delta}$ is pseudo-random under intractability assunption 2.

Theorem 2 If the $S D(\rho, \delta)$ collection is one-way, then $G_{p, \delta}$ is pseudo-random.

Remark: By standard arguments, the samc rcsult cxtcnds to the itcrative gencrator $g_{\rho, \delta}$.

Proof. This proof is inspired from Impagliazzo and Naor [13]. Let $G_{\rho, \delta}$ be the gencrator with matrix $M \in 2^{\lfloor p n\rfloor \times n}$ and weight $w=\lfloor\delta n\rfloor$ as defined in 2.1. If $G_{p, \delta}$ is not pscudo-random, we can build a distinguisher that accepts (i.e. oulputs 1) with a different probability a string generated by $G_{\rho, \delta}$ and a random string. We can then use this distinguisher to predict the inner product of $r$ and $s$ with an advantage better then $\frac{1}{p o l_{y}(n)}$ from given values of $r$ and $G_{p, \delta}(s)$. Using the Goldrcich-Levin theorem, we have a contradiction to the one-wayness of the corresponding $f_{n}^{\rho, \delta}$ function.

When we think of $r$ and $s$ as defining subsets of the matrix columns (the $i$-th column of the matrix is in the subset defined by $r$ (resp. $s$ ) if the $i$-th bit of $r$ (resp. s) is 1), we see that $r \odot s$ is the parity of the intersection. The idea is to use the distinguisher to predict this parity.

The distinguisher is fed with a matrix $M$ and a binary word $t$ of size $\lfloor n\rfloor$. Without loss of gencrality, we can assume that, for infinitely many indices, the distinguisher

- outputs 1 with probability at least $\frac{1}{2}+\frac{1}{p(n)}$ if $t$ is the product of the matrix with a word of weight $w$;

- outputs 1 with probability almost exactly $\frac{1}{2}$ if $t$ is chosen uniformly in $\{0,1\}^{\lfloor\rho n\rfloor}$.

Let $M$ in $\lfloor\rho n\rfloor \times n$ be a matrix, where $r_{i}$ is the $i$-th column of $M: M=$ $\left(c_{i}\right)_{1 \leq i \leq n}$. Given $r=\left(r_{1}, \ldots, r_{n}\right) \in\{0,1\}^{n}$ and $x \in\{0,1\}^{\lfloor p n\rfloor}$ both chosen at random, we construct a new matrix $M_{x}^{r}=\left(c_{i}^{\prime}\right)_{1 \leq i<n}$ such that:

- if $r_{i}=1$, the $i$-th column of $M_{x}^{r}$ is the bitwise xor of $c_{i}$ and $x$ (i.e. $c_{i}^{\prime}=c_{i} \oplus x$ );

- if $r_{i}=0$, the $i$-th column of $M_{x}^{r}$ is $c_{i}$. 
We let $u$ denote a possible output of the pseudo-random generator, i.e $M \cdot s=u$ for some $s$. Let $k=|r \cap s|$ be the number of locations where the bits of $r$ and $s$ are both 1 , and $\epsilon$ be the parity of $k$. As noted above, this is equivalent to saying that $\epsilon$ is the inner product of $r$ and $s$. Let $\epsilon \cdot x=x$, if $\epsilon=1$ and $0^{\lfloor\rho n\rfloor}$ otherwise. Wc observe that the result of $M_{x}^{r}, s$ is $u \oplus \epsilon \cdot x$, as established by the following computation:

$$
\begin{aligned}
M_{x}^{r} \cdot s & =\bigoplus_{c_{i}^{\prime} \in s} c_{i}^{\prime} \\
& =\left(\bigoplus_{c_{i} \in s} c_{i}\right) \oplus\left(\oplus_{c_{i} \in r \cap s} x\right) \\
& =u \oplus \epsilon \cdot x
\end{aligned}
$$

On input $\left(M_{x}^{r}, u \oplus \epsilon \cdot x\right)$, the distinguisher sces exactly the same distribution as on input $(M, u)$. But then, the distinguisher outputs 1 on input $\left(M_{x}^{r}, u \oplus c \cdot x\right)$ with probability at least $\frac{1}{2}+\frac{1}{p(n)}$. On the other hand, if we replace $\epsilon$ by $\bar{\epsilon}$, then, due to the randomness of $x$, the distinguisher is fed with uniformly distributed inputs. The distinguisher outputs 1 with probability $1 / 2$. This lcads us to the following consruction:

Input: $M \in\{0,1\}^{\lfloor p n\rfloor \times n}, u \in\{0,1\}^{\lfloor p n\rfloor}, r \in\{0,1\}^{n}$

- choose a random $x \in\{0,1\}^{\lfloor n !}$ and a random $\theta \in\{0,1\}$ ( $\theta$ is our guess about the inner product)

- feed the distinguisher with $\left(M_{x}^{r}, u \oplus \theta \cdot x\right)$

- if the distinguisher answers 1 , output $\theta$, else output $\bar{\theta}$.

Theorem 3 Our algorithm predicts the inner product with probability at least $\frac{1}{2}+\frac{1}{2 p(n)}$.

Proof. If we have gucssed $\epsilon$, our predictor is correct if the the distinguisher outputs 1 . Wo have seen that this is the case with probability at least $\frac{1}{2}+\frac{1}{p(n)}$.

If wo have not gnessed $\epsilon$ correctly, the prodictor sees the input as a totally random distribution. It then outpuls 1 with probability almost $\frac{1}{2}$. Since both cases happen with probability $\frac{1}{2}$, the overall probability of the algorithm to predict the inner product is at least $\frac{1}{2}+\frac{1}{2 p(n)}$.

\section{Performances}

The computation of the product of the matrix by an vector is done solely by using logical operations like AND, XOR and PARITY, which leads to very fast implementations. The matrix can be either extensively described bit per bit, or defined as the output of a pseudo-random generator so that the matrix description remains small in size. 'This can be done very simply by using a congruential generator with different seeds for each line.

The main bottleneck in the algorithm comes from the sampling algorithm that computes a binary vector of given length and weight. We have to use multiplications and divisions, and that is costly in comparison with the previous operations. However, such computations can be greatly specded up by doing some 
precomputations, like a table of the binomials that will be used. That leaves us with only substractions and comparisons, yelding a very fast computation.

Recent attacks on cryptosystems based on error-correcting codes [5] have shown that a $(512,256)$ random linear code can be decoded up to half its minimum distance 58 . That means that words of weight up to 30 can be found given their syndrome. So we can choosc a weight between 50 and 57 with the assurance of a very good security.

The gain of the generator is $p n-\left[\log _{2}\left(\begin{array}{c}n \\ \lfloor\delta n\rfloor\end{array}\right)\right]$. For a value of $\rho$ fixed to $1 / 2$, we have the following results:

\begin{tabular}{|c|c|c|c|c|c|c|c|}
\hline$n$ & 512 & 512 & 512 & 728 & 728 & 1024 & 1024 \\
\hline$[\delta n]$ & 56 & 55 & 50 & 78 & 71 & 110 & 100 \\
\hline gain & 5 & 8 & 23 & 11 & 32 & 12 & 43 \\
\hline
\end{tabular}

As seen in the table, $n=512$ and $\lfloor\delta n\rfloor=55$ yields one byte per iteration, which looks very attractive.

The scheme as described in section 2 can be improved by precomputing the binomial coefficients. By thus constructing a table of binomials, we get rid of costly multiplications and divisions. The resulting scheme makes only use of comparisons, substractions of $n$-bit numbers and multiplications of a binary matrix with an $n$-bit vector. Note that those operations are very fast since they require only logical or fast instructions. The momory cost of a table of the binomial coefficients for the $(512,256,55)$ scheme is the following: we need $512 \times 55$ entries of size 256 bits, which makes a total of 880 kBytes of memory. This is clearly not an issue for todays computers.

On a SUN Sparc10 station, our implementation using a precomputed binomial table achicves an output rate of 3500 bits per second. RSA with a 512 bit modulus and small cxponent is rated at about $0.005 \mathrm{sec}$ per encryption using the chinese romainders. If we output $\log _{2}(n)=9$ bits per application of the scheme, the output rate is about 1800 bits per second.

\section{Conclusion}

We have shown a construction for a pseudo-random generator with proven security. This gencrator is very efficient and simple, and its implementation is fairly straightforward. We hope that this work will encourage research of alternative solutions to number theory.

\section{Acknowledgements}

We thank Oded Goldreich and Mike Luby for discussions on the subject of this paper. Also, after this paper has been accepted for EUROCRYPT, we were informed that Ramarathnan Venkatesen had considered a similar construction. His work will appear elsewhere. 


\section{A Appendix}

Lemma 1 the product of an $n$-bit integer by an $\log _{2} n$-bit integer is computable in linear time. The same property holds for the division.

Remark: as will be seen from the proof, this actually holds in a model corresponding to random access machines with logarithmic cost. It is unclear that the result carries over in the 'Turing machine model.

Proof. Let $x$ be a $n$-bil integer, $y$ a $\log _{2} n$-bit integer, and $\alpha$ a positive real smaller than $1 / 2$.

We write $x$ and $y$ in basc $2^{t}=2^{\left\lfloor\alpha \log _{2} n\right\rfloor}$ :

$$
x=\sum_{i=0}^{\frac{n}{i}} x_{i} \cdot 2^{t i}, y=\sum_{j=0}^{\frac{\log _{2} n}{t}} y_{j} \cdot 2^{t j}
$$

Then $x \cdot y=\sum_{i, j} x_{i} \cdot y_{j} \cdot 2^{t(i+j)}$.

So the multiplication requires $\left\lceil\frac{n}{i}\right\rceil \times\left\lceil\frac{\log _{2} n}{t}\right\rceil \simeq O\left(\frac{n}{\log _{2} n}\right)$ :

- multiplications of two $t$-bit integers;

- shiftings of $t$-bit integers (the multiplication by $2^{t(i+j)}$ ); additions of two t-bit integers.

Addition and shifting are done in time linear in the length of the input, which is, in our case, in $O\left(\log _{2} n\right)$.

It remains to prove that multiplication of two $\log _{2} n$-bit integers can be done within the same complexity bound. First, we construct once for all a table of all the possible products $x_{i} \cdot y_{j}$. Since $x_{i}$ and $y_{j}$ are in $\left\{0, \ldots, 2^{t}\right\}$, the size of the table is at most $2^{\alpha \log _{2} n} \times 2^{\alpha \log _{2} n}$. Using the quadratic-time multiplication, the table can be computed in time $\left(2^{\alpha \log _{2} n}\right)^{2}\left(\alpha \log _{2} n\right)^{2}$; and since $\alpha<\frac{1}{2}$, this construction is done in time $O(n)$. Table look-up is performed in $O\left(\alpha \log _{2} n+\right.$ $\left.\alpha \log _{2} n+\alpha \log _{2} n\right) \simeq O\left(\log _{2} n\right)$, so the complexity of our multiplication is also $O\left(\log _{2} n\right)$.

Finally, the multiplication of an $n$-bit integer by an $\log _{2} n$-bit integer requires the construction of a table done in $O(n)$ and $O\left(\frac{n}{\log _{2} n}\right)$ operations which are done in $O\left(\log _{2} n\right)$, so that the whole scheme has a complexity of $O(n)$.

\section{References}

1. Alexi, W., Chor, B., Goldreich, O., Schnorr, C. P.: Rsa and rabin functions: certain parts are as hard as the whole. SIAM J. Computing 17 (1988) 194-209.

2. Berlekamp, L. R., McFliece, R. J., van lilborg, H. C. A.. On the inherent intractability of certain coding problems. In IEEE Trans. Information Theory (1978) IEEE pp. 384-386.

3. Blum, L., Blum, M., Shub, M.: A simple unpredictible pseudo-random number generator. SIAM J. Computing 15 (1986) 364-383. 
4. Blum, M., Micali, S.: How to generate cryptographically strong sequences of pseudo-random bits. SIAM J. Computing 13 (1984) 850-863.

5. Canteaut, A., Chabaud, F.: A general improvement of the previous attacks on McEliece's cryptosystem. Unpublished.

6. Chabaud, F.: On the security of some cryptosystems based on error-correcting codes. In Advances in Cryptology: Proc. of EUROCRYPT'94 (1994) LNCS Springer-Verlag.

7. Garey, M. R., Johnson, D. S.. Computers and intractability: a guide to the theory of NP-completencss. W. H. Frceman and Co 1979.

8. Goldreich, O.: Foundations of cryptography (Fragments of a book). Weizmann Institut of Science 1995.

9. Goldreich, O., Krawczyk, H., Luby, M.: On the cxistence of pscudo-random generators. In Proc. 29th Symp. on Foundations of Computing Science (1988) IFEE pp. $12-24$.

10. Goldreich, O., Levin, L. A.: Hard core predicate for any one-way function. In Proc. 21st Symp. on Theory of Computing (1989) ACM press pp. 25-32.

11. Guillot, P.: Algorithmes pour lo codage ì poids constant. Unpublished.

12. Håstad, J., Schrift, A. W., Shamir, A.: 'The discrete logarithm modulo a composite hides $o(n)$ bits. J. of Computing and Systems Science 47 (1993) 376-404.

13. Impaggliazzo, R., Naor, M.: Efficient cryptographic schemes provably as secure. as subset sum. In Proc. 30th Symp. on Foundations of Computing Science (1989) IEEE pp. 236-241.

14. Impagliazzo, R. Levin, L. A., Luby, M.: Pseudo-random generation from any oneway functions. In Proc. 21st Symp. on Theory of Computing (1989) ACM press pp. $12-24$.

15. Levin, L. A.: One-way functions and pseudo-random generators. In Proc. 21st Symp. on Theory of Computing (1985) ACM pp. 363-365.

16. Long, D. L., Wigderson, A.: The discrete $\log$ hides $o(\log n)$ bits. SIAM J. Computing 17 (1988) 363-372.

17. McWilliams, F. J., Sloane, N. J. A.: The theory of error-correcting codes. NorthHolland 1977.

18. Micali, S., Schnorr, C. P... Lfficient, perfect random number generators. In Advances in Cryptology, Proc. of CRYPTO'88 (1988) vol. 576 of LNCS Springer Verlag.

19. Stern, J.:. A method for finding codewords of small weight. In Lecture Notes

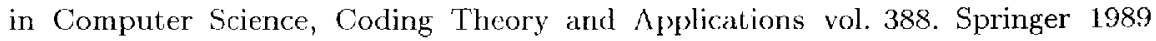
pp. $106-113$.

20. Stern, J.: A new identification scheme based on syndrome decoding. In Advances in Cryplology, Proc. of CRYPTO'93 (1993) vol. 773 of LNCS SpringerVerlag pp. 13-21.

21. Vazirani, U. V., Vazirani, V. V... Efficient and secure pseudo-random sequences from slightly-random sources. In Proc. 25th Symp. on Foundations of Computing Science (1984) IEEE pp. 458-463.

22. Yao, A. C.. Theory and application of trapdoor functions. In Proc. 25th Symp. on Foundations of Computing Science (1982) IEEE pp. 80-91. 